\title{
Inhalt des Jahrgangs 37 / Sommaire du volume 37
}

Erwin H. Ackerknecht, Zur Geschichte der Krebsbehandlung 189

Heinz Balmer, Waadtländer Naturforscher 133

René Bernoulli, Un grand méconnu: Gustave Roussy 34

Urs Boschung, Gustav Adolf Wehrli (1888-1949), Gründer der Medizinhistorischen Sammlung der Universität Zürich

Heinrich Buess, Henry E. Sigerist (1891-1957) und die erste medizinhistorische Konferenz in Pura (Tessin)

Heinrich Buess und Marie-Louise Portmann, Berühmte Schweizer Ärzte 289

Adolf Faller, Elemente einer Wissenschaftslehre und einer Wissenschaftskritik in den Schriften von Niels Stensen (1638-1686)

Othmar Keel, La pathologie tissulaire de John Hunter $\quad 47$

Johann Ulrich Marbach, Domenico Cirillo 257

Markus Mattmüller, Medizingeschichte und allgemeine Historie -

Dialog und Zusammenarbeit auf dem Gebiet der modernen Sozialgeschichte $\quad 62$

Philippe Mudry, Medicus amicus. Un trait romain dans la médecine antique $\quad 17$

Christian Müller, Le Champ-de-l'Air, premier hôpital psychiatrique du Canton de Vaud 28

Marie-Louise Portmann, Relations d'Auguste Tissot (1728-1797), médecin

$\begin{array}{ll}\text { à Lausanne, avec le patriciat bernois } & 21\end{array}$

Marie-Louise Portmann: siehe Buess

Beat Rüttimann, Zur Geschichte der Krüppelfürsorge $\quad 199$

Werner Sackmann, Fleckfieber und Fleckfieberforschung zur Zeit des Ersten Weltkrieges 113

Hans-Konrad Schmutz, Rüdingers «Atlas des peripherischen Nervensystems des menschlichen Körpers» mit Photographien von Joseph Albert 83

Heinz G. Schott, Elemente der Selbstanalyse in den «Studien über Hysterie» 235

Joseph Schürmann-Roth, «Modus et ratio visendi aegros» des Solothurner Arztes Johann Jakob Scharandaeus (1630-1682)

Jean Starobinski, Panorama succinct des sciences psychologiques entre 1575 et $1625 \quad 3$

Gian Töndury, Anatomie in Zürich 271

Albert Wirz, Malaria-Prophylaxe und kolonialer Städtebau: Fortschritt als Rückschritt? $\quad 215$

\section{Kürzere Beiträge}

Heinz Balmer, Naturerkenntnis und Dichtung

Luigi Belloni, Zur Deutung zweier pathologischer Stellen (eine mit Zeichnung) aus der Anatomie von Leonardo: Lungenechinococcus, Halslymphdrüsenverkalkung 139

Klaus-Dietrich Fischer, Der früheste bezeugte Augenarzt des klassischen Altertums $\quad 324$

Huldrych M. Koelbing, Pockennarben und Schönheit 321

Marta Meyer-Salzmann, Die Spitalversorgung im Emmental $\quad 307$

Jean-Pierre Portmann, Notice historique sur les roches moutonnées, polies et striées $\quad 142$

Geburtstage

Heinrich Buess, Paul Röthlisberger zum 70. Geburtstag 
Nachruf

William F.Bynum, Frederick Noël Lawrence Poynter

Berichte

H.M.Koelbing, 27.Internationaler Kongreß für Geschichte der Medizin, Barcelona 1980

Huldrych M. Koelbing, Société Internationale d'Histoire de la Médecine (SIHM)

Buchbesprechungen

Urs-Peter Beerli, Medizinisches in Scheuchzers «Physica sacra» oder Kupferbibel

(Heinz Balmer)

Berner Geographische Mitteilungen (Georges Grosjean, Aus der Geschichte des geographischen Instituts) (Heinz Balmer)

Elisabeth Bennion, Antique Medical Instruments (Urs Boschung)

Manfred Bleuler (Hrsg.), Beiträge zur Schizophrenielehre der Zürcher

Psychiatrischen Universitätsklinik Burghölzli (1902-1971) (Hans H. Walser)

Brian Bracegirdle, A History of Microtechnique (Urs Boschung)

John Cule, Wales and Medicine (Huldrych M. Koelbing)

Oscar Forel, La mémoire du chêne (Hans H. Walser)

Georges Grosjean: siehe Berner Geographische Mitteilungen

Wolfgang-Hagen Hein und Holm-Dietmar Schwarz (Hrsg.), Deutsche Apotheker-Biographie (M-Z) (Gottfried Schramm)

Nicolas de Haller, Albert de Haller, 1708-1777 (Heinz Balmer)

Hans Hoffmann, Gottlob Heinrich Bergmann (1781-1861), ein Hildesheimer Arzt

(Erwin H. Ackerknecht)

Hansruedi Isler, Neurological Sciences in Developing countries (E. H. Ackerknecht)

Othmar Keel, La Généalogie de l'Histopathologie (Erwin H. Ackerknecht)

Erna Lesky, Franz Joseph Gall, 1758-1828, Naturforscher und Anthropologe

(Hans H. Walser)

J.M.López Piñero, F. Bujosa, M.L.Terrada, Clásicos Españoles de la Anatomía

Patológica anteriores a Cajal (Erwin H.Ackerknecht)

Marta Meyer-Salzmann, Michel Schüppach, 1707-1781 (Heinz Balmer) 340

Walter Müri, Der Arzt im Altertum (Huldrych M. Koelbing) 154

David Nachmansohn, German-Jewish Pioneers in Science (Erwin H. Ackerknecht) 334

Beny Olonetzky, Die Sammlung (Urs Boschung)

Heinrich Rodegra, Das Gesundheitswesen der Stadt Hamburg im 19. Jahr-

hundert unter Berücksichtigung der Medizinalgesetzgebung (1586-1818-1900)

(Erwin H. Ackerknecht)

Francis Schiller, Paul Broca, Founder of French Anthropology, Explorer of the Brain (Erwin H. Ackerknecht)

Hedwig Schleiffer, Narcotic Plants of the Old World (Walter Rytz)

Holm-Dietmar Schwarz: siehe Hein

Owen H. Wangensteen und Sarah D. Wangensteen, The Rise of Surgery (B. Rüttimann) 157

Zuschrift 